\title{
Indoor Navigation by Blind People: Behaviors and Challenges in Unfamiliar Spaces and Buildings
}

\author{
Watthanasak Jeamwatthanachai, Mike Wald, Gary Wills \\ School of Electronics and Computer Science University of Southampton, Southampton, UK \\ \{wj1g14, mw, gbw\}@ecs.soton.ac.uk
}

\begin{abstract}
A lot of visually impaired people have suffered from being left out of the activities that can discourage them from going out for any social activities and interactions, which are usually related to navigation. Regardless outdoors, travelling inside public spaces are a different story as many environmental cues cannot be used and have their own set of difficulties. Some technologies have come into play helping these people to have freedom in navigation (e.g., accessible map, indoor navigation systems, wearable computing devices, etc.). However, technologies like accessible maps or indoor navigation system are insufficient to fulfill the independent navigation gap due to additional information is required (obstacles, barriers, and accessibility). To promote the indoor navigation and create better use of technologies for visually impaired people, it is essential to understand the facts, and actual problems that they experience, and what behaviors and strategies they use to overcome any problems, which apparently led to this study. 30 visually impaired people and 15 experts were recruited and asked to give interviews regarding the behaviors, strategies, and challenges found with indoor navigation, especially public spaces e.g., universities, hospitals, malls, museums, and airports. The findings from this present study reveal that navigating inside buildings and public spaces full of unfamiliar features is too difficult to attempt the first time for a number of reasons, reducing their confidence in independent navigation.
\end{abstract}

Keywords: Blindness, Visual impairment, Indoor navigation, Behaviors, Strategies, Wayfinding, Unfamiliar spaces, Public spaces

\section{Introduction}

In 2014, the World Health Organization (WHO) [1] estimated the number of visually impaired people at 285 million worldwide, of which 39 million are blind, and 246 million have low vision. Lack of vision creates difficulties directly affecting most activities, especially those requiring ability to navigate. Being unable to obtain information about where they are or where to go in public buildings such as such as colleges/universities, malls, hospitals, public transportations, and airports causes problems in their wayfinding process discouraging them to go out themselves. The study found $80-90 \%$ of visually impaired people spent their life inside buildings [2].

For visually impaired people, doing such things would not be as easy as sighted people. Challenges and difficulties apply to most of their activities. Travelling without vision is challenging for the congenital blind, adventitious blind and even blindfolded sighted people [3]. Therefore, an orientation and mobility (O\&M) program was created for visually impaired people, teaching safe, efficient, and effective travel skills including how to use common tools e.g. white cane and sensory compensation [4] to sense their surrounding environment as an important part of forming a mental map. Independently walking through the cities or buildings is still difficult as a lot of problems is encountered, for example obstacles, noise, and other barriers which directly affect their daily activities and navigation [5-7].

Visually impaired people have more confidence traveling (outdoors and indoors) with the white cane [5,6], by increasing their perception and familiarity of spaces through the environment cues (e.g. landmarks) they receive when the cane hits something. Traveling inside buildings creates other difficulties since visually impaired people cannot sense and use landmarks to help move around spaces: especially walking through unfamiliar, crowded, and wide-open spaces due to limitation of their sensing environment cues (light, smell, and noise) [4], and randomly-placed obstacles. As a result, visually impaired people may take a long time to familiarize themselves with spaces and to construct a mental map.

When traveling indoors, most of the outdoor challenges are not present, but head-level and trip accidents and even movable objects are still taken into consideration. Learning a new environment like a hospital, mall or large complex building leads to navigation difficulty due to lack of accessibility information and environment cues [5]. It is very hard to decide which way to reach the destination in such a complex crowded environment [8]. A guide 
dog would be a reasonable choice, but sometimes is not permitted, for example, hospitals [9], theaters, and zoos [10]. In the case of visiting an unfamiliar place, the guide dog needs training beforehand to make it familiar with the environment and remember a standard or appropriate route [11].

Some visually impaired people use a variety of accessible maps (e.g. tactile maps) to help navigating indoors and learning the environment beforehand, while others use other assistive technologies e.g. indoor navigation system, wearable computing devices, and mobile devices. Accessible maps play a significant role in improving and accelerating the process of constructing mental maps [12,13], whereas using tactile maps is difficult when not familiar with Braille (Section 3.1) which takes a lot of effort to learn [12]. Information provided in tactile maps is not sufficient in regard to safety, wayfinding, and orientation during navigation indoors [14].

Learning behaviors and challenges visually impaired people found during navigation indoors is essential for design of technologies, which enable independent-indoor navigation in unfamiliar spaces. The aim of this study is to get in-depth understanding of behaviors and strategies by visually impaired people, and investigate challenges found while navigating inside spaces. The study findings are analyzed and classified into 5 categories: (i) use of assistance, (ii) distance estimation, (iii) wayfinding and orientation, (iv) unfamiliar spaces, and (v) obstacle and hazards.

\section{Method}

The purpose of this study was to acquire an in-depth understanding of the indoor navigation behaviors and strategies used by visually impaired people when walking inside buildings full of unfamiliar features. The environment perception of visually impaired people varies, depending on person characteristics [12] such as age, cognitive development, type of blindness (congenital or adventitious), and level of blindness. In this study, 45 participants were recruited and split into 30 visually impaired people and 15 sighted people.

30 visually impaired people were recruited; 10 from the United Kingdom, and 20 from Thailand randomly chosen with evenly distributed numbers of males and females. 10 participants self-reported as congenitally blind, whereas 20 participants were adventitious blind. 22 participants self-reported as severely sight impaired (blind), while 8 participants were sight impaired (partially sighted). The sighted participants were (i) chaperones and O\&M instructors, and (ii) developers and researchers in technical fields: human-computer interaction, accessibility, assistive technology, and interior design for elderly and disabled people.

Both groups completed questionnaires: Eighteen questions (10 closed and 8 open) were listed for visually impaired people, while 18 questions ( 9 closed and 8 open) were used for sighted people. All questions were split into five categories regarding indoor navigation by people with visual impairment (see Appendix A). Semi-structured interviews were conducted for people with visual impairment however, the findings from both groups were used in terms of complementarity, clarity, and elaboration of the findings.

\section{Findings}

Analysis of findings classified into five sections showed that visually impaired people experience a lot of problems navigating inside the buildings and public spaces e.g. universities, hospitals, malls, museums, and airports.

- Section 3.1 describes common tools visually impaired people use, including restrictions of the use of assistance in particular places.

- Section 3.2 discusses how visually impaired people estimate distance in unfamiliar spaces.

- Section 3.3 describes wayfinding strategies and how people find their orientation in unfamiliar spaces

- Section 3.4 discusses problems and challenges found in the public spaces including other four type of buildings were consequently found during the interviews.

- $\quad$ Section 3.5 discusses obstacles and hazards discovered while navigating indoors.

3.1. Use of Assistance 


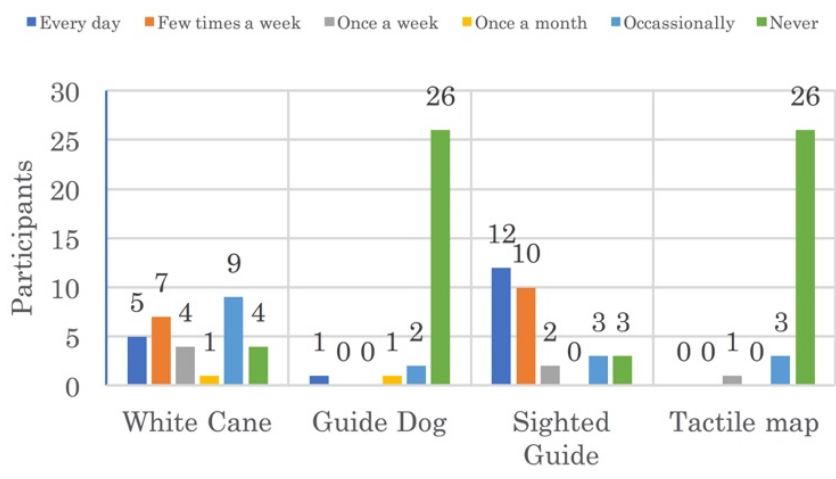

Figure 1. Survey of Use of Assistance

Participants were asked if they had received Orientation and Mobility (O\&M) training or not, and what types of assistance they usually use for daily activities. 19 participants (63\%) indicated they received training while $11(34 \%)$ did not giving reasons such as another physical challenge and age-affect learning ability. Figure 1 shows what assistance has been used to navigate indoors and how frequently. Therefore, the various assistances are ranked using the Borda Scoring Rule [15], shown in Table 1. Consequently, the white cane and a sighted guide are the most promising assistance used to perform daily activities, with the preference for a sighted guide as the first choice while the white cane is used once people are familiarized with spaces and buildings by repeatedly visiting there a number of times. This contradicts the common belief of sighted people that the white cane is the preferred method of traveling, the data show that people use a mixture of methods when navigating in an unfamiliar spaces and buildings.

\begin{tabular}{|l|c|c|c|c|}
\hline \multirow{2}{*}{ Type of Assistance } & \multicolumn{2}{|c|}{ Visually Impaired People } & \multicolumn{2}{c|}{ Sighted People } \\
\cline { 2 - 5 } & BSR & Ranking & BSR & Ranking \\
\hline Sighted Guide & $\underline{109}$ & $\underline{1}$ & 49 & 2 \\
\hline White Cane & 76 & 2 & $\underline{63}$ & $\underline{1}$ \\
\hline Tactile Map & 9 & 3 & 13 & 3 \\
\hline Guide Dog & 6 & 4 & 13 & 3 \\
\hline
\end{tabular}

People with visual impairment suggested some challenges the white cane cannot overcome such as detecting slippery floor caused by water or fabric dropped on the floor, as well as body-level and head-level obstacles they run into inside buildings. In some building, stepping down from a change in level to circulations (e.g. corridor and pathway) is especially challenging, as they could collide with some head-level obstacle. As a result, a sighted guide is the most popular assistance ahead of a white cane, while a guide dog and tactile map were not chosen for the following reasons.

A guide dog is not affordable as it costs up to $£ 27,300$ for the special training until it is ready for people with visual impairment [9], and Thailand has no guide dog training center. However, to use a guide dog in unfamiliar spaces it needs to be trained beforehand to memorize the standard and safe route, which usually takes 2-3 or more times walking through the building until it can remember the route. This can be undertaken by the guide dog trainers, but one participant said that a guide dog is not permitted in Islamic religious spaces because the dog is used for hunting, not as a pet or helper.

Likewise, a tactile map is not widely used since it is not so easy to use, and interpret its contents. Most said that the tactile map required a lot of effort to understand the contents provided. 4 (13\%) participants had used a tactile map to find their way to a destination. 2 participants had successfully reached the destination, while the other 2 did not as the content provided was confusing and non-standard which led to miscalculating the distance.

\subsection{Distance Estimation}




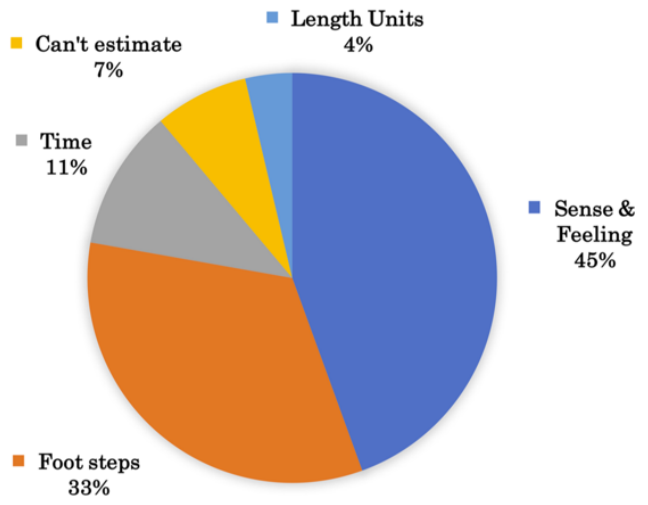

(a) Visually Impaired People

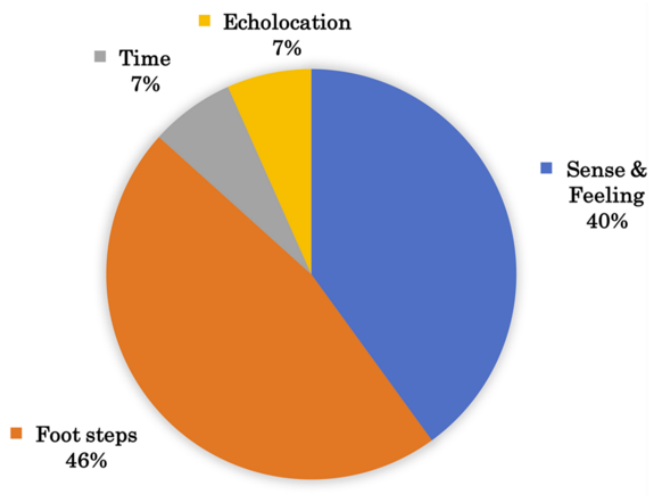

(b) Sighted People

Figure 2. Distance Estimation Survey

Many strategies have been used in distance estimation in both familiar and unfamiliar spaces (see Figure 2). There are different points of view between people with visual impairment and sighted people. The sighted suggested walking in the unfamiliar spaces, while people with visual impairment do not know the features inside the spaces, so a strategy of counting footsteps is appropriate instead of the use of landmarks as one of a combination of strategies. On the other hand, people with visual impairment argued that the counting footsteps is sometimes difficult and takes a lot of mental effort and is easily distracted if walking into obstacles (e.g. furniture and landmarks), or into people, especially in unfamiliar spaces with full of crowds and noise. As a matter of fact, about half of participants rely on their sense and feeling, focusing on the use of environment information to estimate distance, e.g. smell, light, floor texture, and landmarks. However, not all of participants can estimate distance especially those born blind who have never seen the world.

\subsection{Wayfinding and Orientation when travelling in unfamiliar spaces}

Most navigation problems in unfamiliar spaces resolve into three challenges: find the current location, find the way to a destination, and find and maintain orientation. This suggests what kind of information people are looking for when entering unfamiliar spaces.

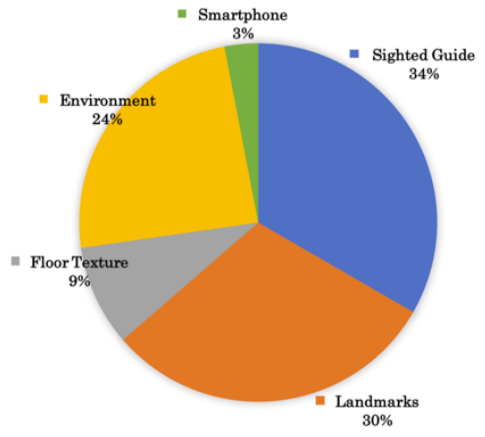

(a) Find Current Location

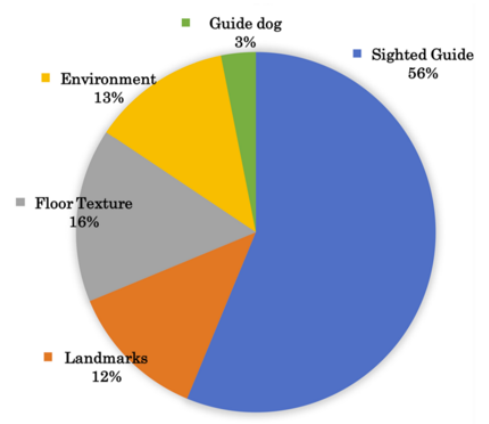

(b) Find Way to Destination

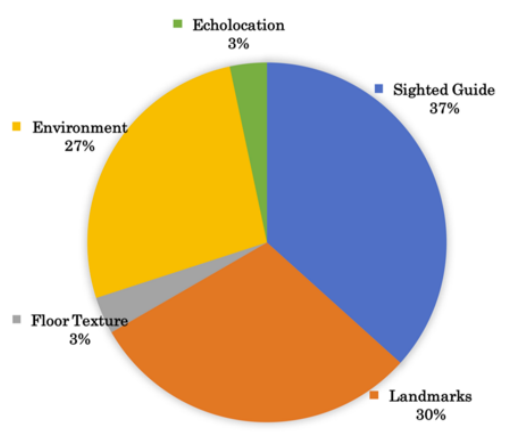

(c) Find Orientation

Figure 3. Wayfinding and Orientation Survey

In Figure 3, it shows the findings from the survey of visually impaired people, which suggests that when they visit unfamiliar spaces, they first need a sighted guide in order to learn and familiarize themselves with the spaces, rather than use landmarks to find location and orientation. Once they have learned the spaces, they will use sense and feeling (e.g. landmarks and environment information) to navigate themselves.

For finding the current location, participants usually estimate where they are from three different sources: $34 \%$ sighted guide, $30 \%$ landmarks, and 24\% environment cues (light, noise, and odor), while using a smartphone is rare due to its limitation and accuracy of estimating location indoors. To navigate to the destination, people asked 
a sighted guide to take them there, or asked for instructions and walked themselves using three simultaneous clues: floor texture, environment cues, and landmarks.

For orientation, visually impaired people usually get confused by asking local people or a sighted guide. Since they are unfamiliar with the space, visually impaired people are not able to use any source of information in the area, e.g. landmarks on which the sighted usually rely, in order to estimate their location and orientation. One participant said that to find his orientation while navigating in his home, he has drawn an image of the relationships between each landmark and other landmarks, while another one has employed echolocation in wayfinding to detect her orientation.

\subsection{Unfamiliar Spaces}

This section discusses the level of difficulty of, and the level of confidence required in, unfamiliar spaces, e.g. university, hospital, department store, museum, and airport. The findings show that most participants (up to $70 \%$ ) have not usually visited such venues due to the physical challenges, where they need assistance from a sighted guide or a local person. Most participants agreed navigating inside the buildings listed in Figure 4 was too difficult by themselves for the following reasons.

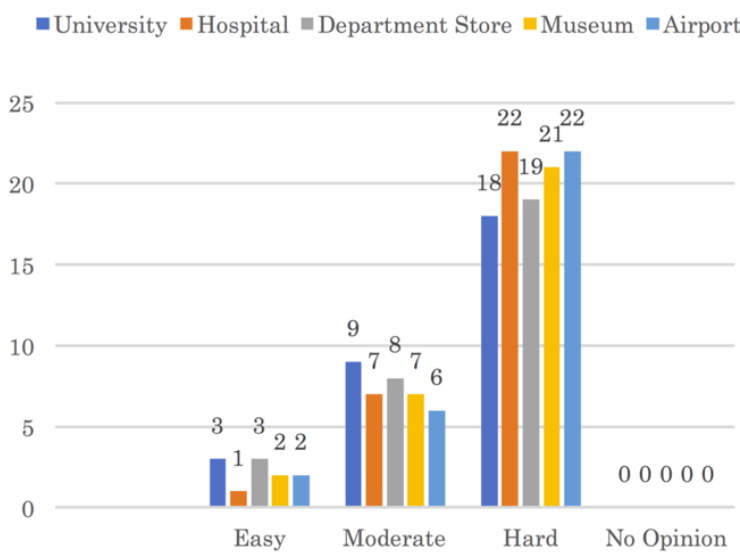

(a) Level of Difficulty

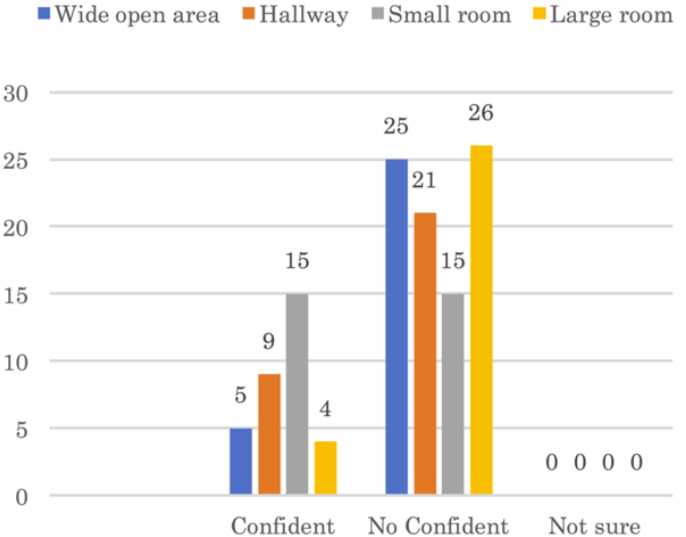

(b) Confidence

Figure 4. Unfamiliar Spaces Survey

Universities, Complex and medium to large buildings.

Colleges

Most participants aged between 18-29 said that navigating within buildings of colleges and universities is either medium or hard, depending on the size of population inside the buildings, and type of building. For example, engineering buildings are usually complex and have a lot of engineering equipment and furniture, which are usually relocatable and able to cause them any harm.

Hospitals,

Busy, noisy, complex and large sized buildings.

Public

Healthcare

Up to 22 of participants (73\%) agreed walking inside a hospital is very difficult due to population size of e.g. patients, nurses, doctors, and visitors, and a lot of obstacles, e.g. hospital beds and furniture. Most participants were distracted by the noise and the many obstacles surrounding them, which therefore made them lose their way, and also lose orientation since they cannot use any landmark and environment information.

Shopping Shopping Mall: Busy, large, wide-open and crowded buildings.

Malls, Department Store: Busy, large, but well-organized buildings.

Department Supermarket: Small to medium buildings

Stores,

Even though the buildings are well-structured, participants still found it difficult to walk by 
Supermarkets themselves. 18(60\%) agreed shopping is challenging due to obstacles, e.g. people and trolleys, and problems in reading information, e.g. price and product name. Most shops do not provide any accessibility information on the label of the products, e.g. Braille or audio feedback, but some, like medicine, are embossed with Braille. This is useful, but Braille cannot be put on every package due to its limitation and cost. As a result, visually impaired people cannot find what they are looking for.

Museums, Exhibitions

Airports
Complex, well-organized and medium to large buildings.

This type of building is usually full of exhibitions, e.g. antique statues or paintings, which are valuable. 22 of the participants (73\%) said that they need to be careful when walking inside this type of building since they could damage the exhibits. The signage cannot be seen or read. To prevent physical contact, most buildings usually set up a colored line (on the floor) or a rope to indicate that the area behind the line is prohibited. This can be a problem since visually impaired cannot see the line when walking in a museum. Participants suggested a rope, edge, and handrail are useful, possibly, illuminated which provides good contrast for partially sighted people.

Busy, noisy, complex and large buildings. Some areas are wide-open.

These buildings are typically large and full of people walking around, and airport furniture, e.g. check-in counters and seating, which are all counted as obstacles. Most participants (73\%) said walking inside an airport alone is very difficult, since they often bump into people. Noise is also an obstacle, making them lose their orientation. Walking in the wide-open areas is still difficult as it makes it easier to get lost, since they cannot find landmarks in the noisy and busy environment.

To reach the Gate is still difficult due the complexity of the buildings and long distances they need to walk, although they can request support services. Furthermore, escalators must be used, which most people with visual impairment are afraid of because they do not know where the steps are, and the edge of the steps is not high contrast which is dangerous if they trip and fall.

Considering these five types of building, there are four types of spaces inside the building and public space whose difficulty in navigating need to be considered separately: wide-open area, hallway, small room, and large room. From the survey, 21 to 26 participants (70-87\%) said they are not confident in navigating through wide-open areas, hallways and large rooms, as it is too difficult to navigate independently. Most participants got lost usually while just standing or navigating through wide-open areas. Most said that there are not many landmarks they can use to help them detect their orientation, such as wall, door, curb, edge, and surface. With noise and people walking about, a lot of distractions to visually impaired people are created.

Large room refers to auditoriums, stadiums, concerts, theaters, and halls. Up to 26 participants (87\%) said that large rooms are usually full of (auditorium) chairs and tables, and hallways are too wide to navigate by themselves. The problem is that, when they do attend events, e.g. concerts, movie theaters, or conferences, they could not find their seat as it has no Braille indication. As with stairs where tread depth and rise height are not standard, the dimension of chairs is not standard or the offset space between each row in the seating area.

For hallways and corridors, some of the participants are confident $(30 \%)$ in navigating them, while others are not $(70 \%)$ because some hallways and corridors are too wide, and that makes them afraid of walking alone. When they tried to walk along the wall, there was furniture installed in the space, and obstacles attached on the wall, but too high to be detected by white cane, e.g. wall-mounted telephone and fire extinguisher. Two participants suggested that some visually impaired people have used the edge of a door to detect their orientation in order to find their way. However, this edge is usually sharp and sometimes harm people encountering it.

For the small rooms, half of the participants said that they had a lot of confidence, since they can find landmarks easier than other types of space, even though the settings are changing all the time. The other half countered that 
it is still difficult to navigate these spaces if they are not familiar with them. This situation is similar to walking in museums. They are afraid that they could damage things inside the room.

In addition to the unfamiliar spaces, however, the findings also suggested a further four types of buildings that are usually visited for daily activities: train stations, libraries, canteens/cafeterias, and auditoriums/halls.

Train Stations Noisy, busy, small to medium buildings, and dangerous spaces.

The first is noise that distracts them a lot while they attempt to find their orientation and gather train information. In this type of building it is hard to find a landmark where a crowd of people gather in the day time. The second problem is a gap between the trains and the platforms. All the partially sighted participants said that they are afraid of stepping up to the train because they cannot find the edge, even though it is usually indicated in yellow color with high contrast.

Libraries Well-organized, busy, and small to medium buildings, but too silent.

This type of building is usually silent and full of furniture, e.g. chairs, tables, bookshelves, but well-organized. Most partially sighted participants said that it is very difficult to find a book themselves due to blurry vision which requires using a magnifying glass or device in order to identify the name of the book. Most books do not have Braille embossed on their covers. Looking for books on your own becomes impossible. Likewise, all the partially sighted participants said that some libraries have automatic sliding door systems installed at the entrance or elsewhere. This type of door usually has no any warning signs saying that it is glass and transparent.

This could be a problem to people who are partially sighted because they cannot distinguish between the door and empty space. Some buildings, on the other hand, have put warning label on the doors, but can be hardly seen because it is not sufficiently high contrast. In addition, this type of door sometimes does not work due to some technical difficulty, e.g. sensors or mechanical issues, and could harm its users.

Canteens, Canteens and cafeterias are usually filled with tables and chairs which can be relocatable Cafeteria within the space. This problem sometimes affects visually impaired when visiting, depending on the number of people in the area. For example, lunch time at the universities and shopping centers are usually filled with crowds and noise.

Auditoriums, Busy, large buildings.

Stadiums,

Concerts,

Theaters,

Halls

Walking inside this type of building is sometimes difficult, depending on situations, events and number of people. One participant said that it was very hard, when she attended the concert, to find her seat even though accompanied by friends. Also, this type of building often does not usually provide any labelling and accessibility for disabled people.

\subsection{Obstacles and Hazards in Indoor Navigation}

Obstacles and barriers are something that people with visual impairment face every day. Most participants reported being hit by obstacles that can cause them to lose their body-balance and orientation, which is most important while navigating. In this section, the finding shows how often most of visually impaired people experienced hitting various obstacles, see Figure 5. 


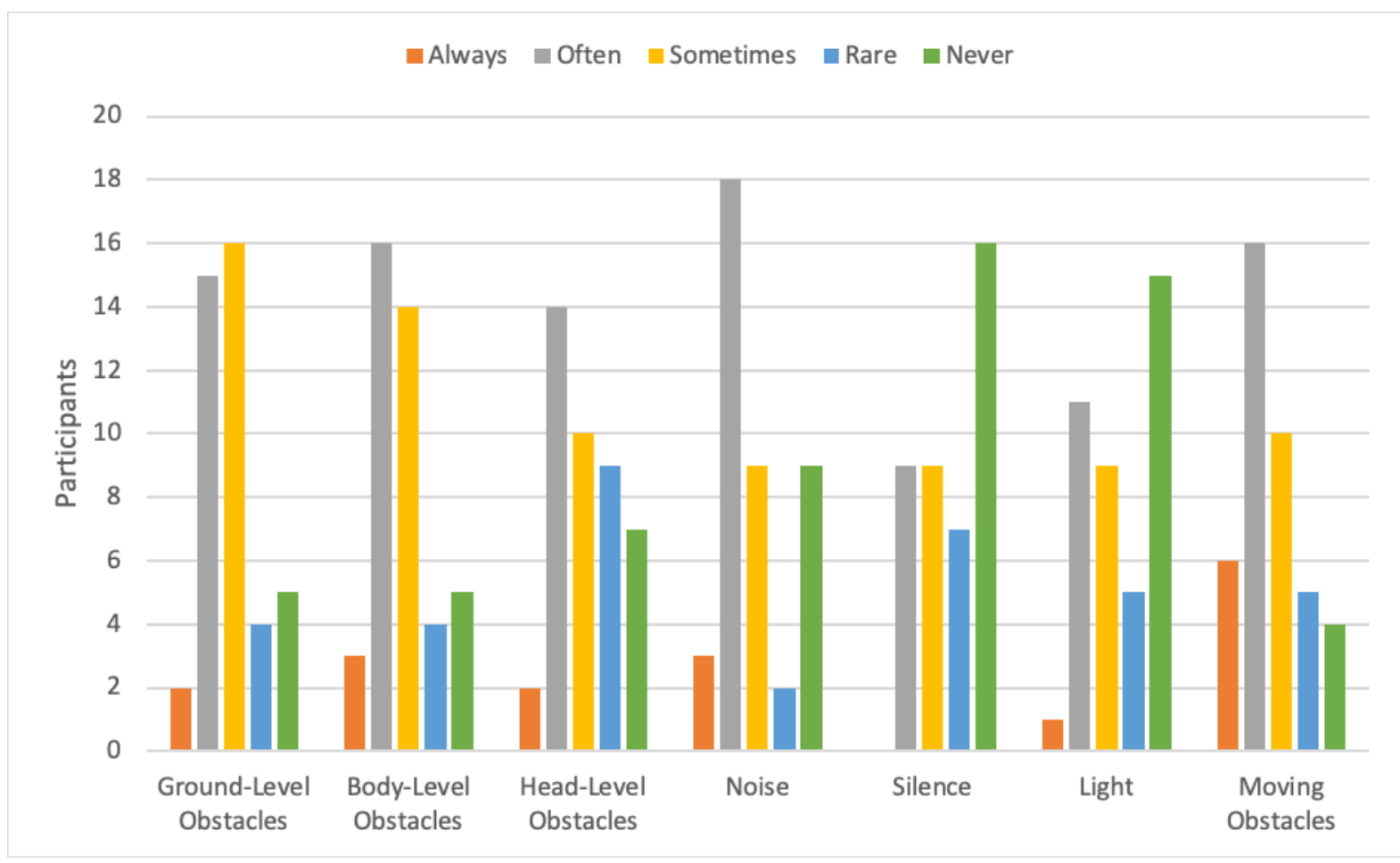

Figure 5. Obstacles Experience Survey

It is clear that noise is the most impact factor they face, while silence is the least. In a wide-open area, too much noise creates a lot of distraction, which findings in a loss of orientation. This situation usually happens when the space is full of crowds, e.g. wide-open area in a department store. On the other hand, a too quiet space tends to panic people with visual impairment. They may think that the direction they are walking in is wrong. This situation is serious. People may end up injured if they are walking inside a building where some area is being renovated, and warning notices are not provided.

Most participants said that the majority of obstacles they hit are at body-level and head-level, e.g. wall-mounted furniture and objects, and hanging labels, since they cannot detect them with a white cane or a guide dog, which are only able to detect ground-level obstacles. In addition, the white cane cannot detect some slippery floor hazards, especially a wet floor or the objects that make the floor slipperier when dropped on the floor, e.g. fabric. However, moving obstacles e.g. people and trolleys, were also mentioned as the obstacles that up to $70 \%$ of people with visual impairment face.

This study also found that light is useful and important to partially sighted people. People have used the light as a helper to detect distance and orientation while navigating inside buildings. However, in some areas, if the light is too bright and illuminates the eyes, it causes temporary blindness.

\section{Discussion}

In this study, 45 people (30 people with visual impaired, and 15 sighted people) were interviewed using the questionnaire with 5 categories (18 questions, see Appendix A). This resulted in 45 hours of audio recording which gave a rich set of data for analysis. The finding of this study is considered the important since there is no previous study on indoor navigation behavior by blind people in unfamiliar spaces and buildings. Thus, the findings of the present study will be particularly important for building and interior designers to make better and friendly environment for people with visual impairment. Moreover, the findings can be useful for O\&M instructors and specialists to improve O\&M courses and training, and of course researchers who work in the field of assistive technology for better indoor navigation for blind people.

The findings of our study indicated that visually impaired people have experienced the challenges when walking inside unfamiliar spaces, where a common tool like the while cane cannot help them navigate independently. Most 
of the participants cannot navigate in those spaces at the first attempt. Therefore, they need to be accompanied by a sighed guide, in order to learn and familiarize themselves with the space before independent navigation with a white cane or other assistance.

While visually impaired people have used the sense and feeling instead of counting footsteps to find their location and orientation, the findings suggested that employing a combination of strategies for navigation instead of using one particular strategy creates confidence in navigation in familiar spaces. For example, one has simultaneously used their sense and feeling while counting footsteps during indoor navigation. Another used a timing strategy together with their sense and feeling. For unfamiliar spaces, however, people with visual impairment usually rely on a sighted guide and landmark and environment information.

\section{Conclusion}

Outdoors, visually impaired people can sense and use the environment cues from the white cane, while inside public spaces many of environment cues cannot be used and has its own set of difficulties. Some technologies help these people to have freedom in navigation need additional information (obstacles, barriers, and accessibility), which led to this study for the promotion of navigation by visually impaired people.

The findings revealed that navigating inside buildings and public spaces full of unfamiliar features is too difficult to attempt the first time for a number of reasons, reducing confidence in independent navigation. Navigation inside buildings by visually impaired people is a challenge as it takes a long time to become familiar with spaces which leads to the need of sighted people's assistance but is a problem regarding distance estimation, and instruction to the destination.

Many difficulties are experienced by visually impaired people while inside the public spaces full of unfamiliar features, for example obstacles, barriers and accessibility information, that have made them unable to navigate safely. To overcome that, the use of indoor landmarks was found to be significantly useful to visually impaired people. Not only landmarks have been used, the environment cues (e.g. light, smell, and noise) are also used in the wayfinding and mental map construction processes.

The findings of this study investigating the problems and challenges experienced by visually impaired people during the indoor navigation, and what kind of behaviors and strategies that visually impaired people use to overcome the problems can be useful for further developments such as indoor navigation systems, wearable computing devices, and mobile devices as well as for O\&M specialists and instructors, in constructing a spatial representation [16].

\section{Appendix A:}

\begin{tabular}{|c|c|c|c|}
\hline$\#$ & Section & People with visual impairment & Sighted people \\
\hline 01 & \multirow{2}{*}{$\begin{array}{l}\text { Use of } \\
\text { Assistanc } \\
\text { e }\end{array}$} & $\begin{array}{l}\text { Did you attend a mobility training course, e.g. } \\
\text { orientation and mobility? }\end{array}$ & $\begin{array}{l}\text { Is a mobility training course (e.g. orientation and } \\
\text { mobility) important to visually impaired and blind } \\
\text { people? }\end{array}$ \\
\hline 02 & & $\begin{array}{l}\text { Which assistance do you use when you navigate } \\
\text { inside buildings by yourself? How often do you use } \\
\text { the assistance for your daily routines? }\end{array}$ & $\begin{array}{l}\text { Which assistance do visually impaired and blind } \\
\text { people use when they navigate inside buildings by } \\
\text { themselves? How often do they use this assistance? }\end{array}$ \\
\hline 03 & $\begin{array}{c}\text { Distance } \\
\text { Estimatio } \\
\mathrm{n}\end{array}$ & $\begin{array}{l}\text { How do you estimate the distance while navigating } \\
\text { inside buildings by yourself? }\end{array}$ & $\begin{array}{l}\text { How do visually impaired and blind people estimate } \\
\text { the distance while navigating inside buildings by } \\
\text { themselves? }\end{array}$ \\
\hline 04 & \multirow{4}{*}{$\begin{array}{l}\text { Wayfindi } \\
\text { ng and } \\
\text { Orientati } \\
\text { on when } \\
\text { travelling } \\
\text { in } \\
\text { unfamilia } \\
\text { r space }\end{array}$} & $\begin{array}{l}\text { How often do you navigate by yourself inside } \\
\text { unfamiliar spaces, e.g. university, hospital, } \\
\text { department store, museum, and airport? }\end{array}$ & $\begin{array}{l}\text { How often do visually impaired and blind people } \\
\text { navigate by themselves inside unfamiliar spaces, e.g. } \\
\text { university, hospital, department store, museum, and } \\
\text { airport? }\end{array}$ \\
\hline 05 & & $\begin{array}{l}\text { What is the level of difficulty to navigate in } \\
\text { university, hospital, department store, museum, and } \\
\text { airport? }\end{array}$ & $\begin{array}{l}\text { While navigating inside buildings by visually } \\
\text { impaired and blind people, what is the level of } \\
\text { difficulty to navigate in university, hospital, } \\
\text { department store, museum, and airport? }\end{array}$ \\
\hline 06 & & $\begin{array}{l}\text { Do you have confidence to navigate inside buildings } \\
\text { by yourself through wide-open, hallway, room, large } \\
\text { room? }\end{array}$ & $\begin{array}{l}\text { Do visually impaired and blind people have } \\
\text { confidence to navigate by themselves through wide- } \\
\text { open, hallway, room, large room? }\end{array}$ \\
\hline 07 & & $\begin{array}{l}\text { Inside unfamiliar spaces inside buildings, how do } \\
\text { you know where you are? }\end{array}$ & $\begin{array}{l}\text { In unfamiliar spaces inside buildings, how do } \\
\text { visually impaired and blind people know where they } \\
\text { are? }\end{array}$ \\
\hline
\end{tabular}




\begin{tabular}{|c|c|c|c|}
\hline 08 & \multirow[t]{5}{*}{$\begin{array}{l}\text { Unfamili } \\
\text { ar spaces }\end{array}$} & $\begin{array}{l}\text { Inside unfamiliar spaces inside buildings, how do } \\
\text { you reach your destination? }\end{array}$ & $\begin{array}{l}\text { Inside unfamiliar spaces inside buildings, how do } \\
\text { visually impaired and blind people reach your } \\
\text { destination? }\end{array}$ \\
\hline 09 & & $\begin{array}{l}\text { How can you find your orientation or the direction } \\
\text { you are heading to? }\end{array}$ & $\begin{array}{l}\text { How can you find your orientation or the direction } \\
\text { visually impaired and blind people are heading to? }\end{array}$ \\
\hline 10 & & $\begin{array}{l}\text { What landmarks are you looking for in order to } \\
\text { learn or to help you to navigate by yourself through } \\
\text { unfamiliar spaces? }\end{array}$ & $\begin{array}{l}\text { What landmarks are visually impaired and blind } \\
\text { people looking for in order to learn or to help you to } \\
\text { navigate by yourself through unfamiliar spaces? }\end{array}$ \\
\hline 11 & & $\begin{array}{l}\text { Have you ever asked anybody (e.g. reception and } \\
\text { local people) for directions or instructions to reach } \\
\text { the destination? }\end{array}$ & \\
\hline 12 & & $\begin{array}{l}\text { Have you ever found any directions or instructions } \\
\text { from local people has been confusing or ambiguous? }\end{array}$ & $\begin{array}{l}\text { Have visually impaired and blind people ever found } \\
\text { that directions or instructions from local people have } \\
\text { been confusing or ambiguous? }\end{array}$ \\
\hline 13 & \multirow{6}{*}{$\begin{array}{c}\text { Obstacles } \\
\text { and } \\
\text { Hazards } \\
\text { in Indoor } \\
\text { Navigatio } \\
n\end{array}$} & $\begin{array}{l}\text { Have you experienced any problems or challenges } \\
\text { while navigating by yourself inside buildings? For } \\
\text { example, ground/body/head-level obstacles, noise, } \\
\text { silent, or light. }\end{array}$ & $\begin{array}{l}\text { Have visually impaired and blind people experienced } \\
\text { any problems or challenges while navigating inside } \\
\text { buildings? For example, ground/body/head-level } \\
\text { obstacles, noise, silent, or light. }\end{array}$ \\
\hline 14 & & $\begin{array}{l}\text { By navigating inside buildings by yourself, have you } \\
\text { ever experienced hitting or being hit by the following } \\
\text { obstacles? }\end{array}$ & $\begin{array}{l}\text { When navigating unfamiliar spaces inside buildings, } \\
\text { do visually impaired and blind people experience a } \\
\text { collision by the following obstacles? }\end{array}$ \\
\hline 15 & & $\begin{array}{l}\text { What obstacles can you detect by your assistance } \\
\text { (e.g. white cane, guide dog, tactile map)? }\end{array}$ & $\begin{array}{l}\text { What obstacles can they detect by their assistance } \\
\text { (e.g. white cane, guide dog, tactile map)? }\end{array}$ \\
\hline 16 & & $\begin{array}{l}\text { What obstacles are you not able to detect by your } \\
\text { assistance (e.g. white cane, guide dog, tactile map)? }\end{array}$ & $\begin{array}{l}\text { What obstacles are they not able to detect by their } \\
\text { assistance (e.g. white cane, guide dog, tactile map)? }\end{array}$ \\
\hline 17 & & $\begin{array}{l}\text { While navigating inside buildings, how do you sense } \\
\text { or know any dangers in your way? }\end{array}$ & $\begin{array}{l}\text { While navigating inside buildings by visually } \\
\text { impaired and blind people, how do they perceive any } \\
\text { dangers in their way? }\end{array}$ \\
\hline 18 & & $\begin{array}{l}\text { Have you ever found that using stairs, escalators, or } \\
\text { elevators is difficult and dangerous? }\end{array}$ & $\begin{array}{l}\text { Have they ever found that using stairs, escalators, } \\
\text { or elevators is difficult and dangerous? }\end{array}$ \\
\hline
\end{tabular}

\section{References}

1. Centre, W.M. Visual impairment and blindness. http://www.who.int/mediacentre/factsheets/fs282 /en/, 2014. [Online; accessed 16-May-2016].

2. Li, K.J., Lee, J. Indoor spatial awareness initiative and standard for indoor spatial data. Proceedings of IROS 2010 Workshop on Standardization for Service Robot, 2010, Vol. 18.

3. Picinali, L., Afonso, A., Denis, M., Katz, B.F. Exploration of architectural spaces by blind people using auditory virtual reality for the construction of spatial knowledge. International Journal of Human-Computer Studies 2014, 72, 393-407.

4. Zaira Cattaneo, T.V. Blind Vision: The Neuroscience of Visual Impairment 2011. pp. 1-285.

5. Williams, M.A., Hurst, A., Kane, S.K. Pray before you step out: describing personal and situational blind navigation behaviors. Proceedings of the 15th International ACM SIGACCESSConference on Computers and Accessibility. ACM, 2013, p. 28.

6. Williams, M.A., Galbraith, C., Kane, S.K., Hurst, A. just let the cane hit it: how the blind and sighted see navigation differently. Proceedings of the 16th international ACM SIGACCESS conference on Computers \& accessibility. ACM, 2014, pp. 217-224.

7. Zeng, L. A Survey: Outdoor Mobility Experiences by the Visually Impaired. Mensch und Computer 2015Workshopband 2015.

8. Ganz, A., Schafer, J., Gandhi, S., Puleo, E., Wilson, C., Robertson, M. PERCEPT indoor navigation system for the blind and visually impaired: architecture and experimentation. International Journal of Telemedicine and Applications 2012, 2012, 19.

9. Guidedogs.org.uk. Are dogs allowed everywhere? - All Access Areas | Guide Dogs. https://goo.gl/ gxQp39, 2016. [Online; accessed 5-May-2016].

10. Guidedogs.org.au. Frequently Asked Questions - GuideDogs SA/NT. https://www.guidedogs.org. $\mathrm{au} /$ frequently-asked-questions, 2016. [Online; accessed 5-May-2016].

11. Kulyukin, V., Gharpure, C., Nicholson, J., Pavithran, S. RFID in robot-assisted indoor navigation for the visually impaired. Proceedings of 2004 IEEE/RSJ International Conference on Intelligent Robots and Systems, 2004. (IROS 2004). IEEE, 2004, Vol. 2, pp. 1979-1984.

12. Martins, L.B., Lima, F.J., others. Analysis of Wayfinding Strategies of Blind People Using Tactile Maps. Procedia Manufacturing 2015, 3, 6020-6027.

13. Schinazi, V.R., Thrash, T., Chebat, D.R. Spatial navigation by congenitally blind individuals. Wiley 
Interdisciplinary Reviews: Cognitive Science 2016, 7, 37-58.

14. Papadopoulos, K., Charitakis, K., Kartasidou, L., Kouroupetroglou, G., Gumus, S.S., Stylianidis, E., Stiefelhagen, R., Müller, K., Yilmaz, E., Jaworek, G., others. User requirements regarding information included in audio-tactile maps for individuals with blindness. International Conference on Computers Helping People with Special Needs. Springer, 2016, pp. 168-175.

15. Shoham, Y., Leyton-Brown, K. Multiagent systems: Algorithmic, game-theoretic, and logical foundations, Cambridge University Press, 2008.

16. Jeamwatthanachai, W., Wald, M., \& Wills, G. (2017). Map Data Representation for Indoor Navigation by Blind People. International Journal of Chaotic Computing, 4(1), 70-78. 